\title{
Nanoslot laser
}

\author{
Shota Kita, ${ }^{\text {a) }}$ Shoji Hachuda, Kengo Nozaki, and Toshihiko Baba ${ }^{\text {b) }}$ \\ Department of Electrical and Computer Engineering, Yokohama National University, 79-5 Tokiwadai, \\ Hodogayaku, Yokohama 240-8501, Japan and Core Research for Evolutional Science and Technology, Japan \\ Science and Technology Agency, 5, Sanbancho, Chiyodaku, Tokyo 102-0075, Japan
}

(Received 21 June 2010; accepted 3 October 2010; published online 21 October 2010)

\begin{abstract}
We propose and demonstrate photonic crystal nanolaser with a nanoslot. Using high-aspect etching process, we succeed in fabricating a 30-nm-wide nanoslot device and room temperature lasing in both air and liquids. As an index sensor, it exhibits a high sensitivity of $410 \mathrm{~nm}$ per refractive index unit, as well as low temperature dependence in water. These behaviors and theoretical analysis suggest that the mode is strongly localized in the nanoslot. This device will be effective for enhancing light-matter interaction in cavity quantum electro dynamics, nonlinear optics, and biosensing. () 2010 American Institute of Physics. [doi:10.1063/1.3505139]
\end{abstract}

Microcavities consisting of high-index-contrast (HIC) photonic crystals $(\mathrm{PCs})$ and whispering gallery structures achieve a high $Q$ and small modal volume $V_{\mathrm{m}}$ of the order of a cubic wavelength in a medium, $(\lambda / n)^{3}$. Further reduction in $V_{\mathrm{m}}$ is crucial for efficient light-matter interaction in cavity quantum electro dynamics and nonlinear optics. ${ }^{1-4}$ For this purpose, a one-dimensional PC cavity with a nanoslot (NS) has been proposed, which strongly confines a cavity mode in the NS using large discontinuities in modal electric field at HIC boundaries. In this case, $V_{\mathrm{m}}$ is reduced dramatically to $0.0053\left(\lambda / n_{\text {air }}\right)^{3}$, where $n_{\text {air }}$ is the index of air. ${ }^{5}$ Such NS is useful not only for above applications but also for sensing liquids and bio-molecules, ${ }^{6,7}$ and trapping nanoparticles. ${ }^{8}$ Previously, we reported the room-temperature continuouswave lasing in HO PC nanolaser, which consists only of the shift of some airholes and achieves a high passive $Q$ of $10^{5}$ and a small $V_{\mathrm{m}}$ of $0.15(\lambda / n)^{3}$. ${ }^{9}$ We also demonstrated the high-resolution sensing of liquids using this nanolaser. ${ }^{10}$ In this study, we aim at a further reduction in $V_{\mathrm{m}}$ and an increase in sensitivity to the environmental index, by introducing a NS into the nanolaser. In general, the NS degrades $Q$ and hence the spectral linewidth and sensing resolution. However, unlike passive cavities, in nanolasers, laser operation narrows the linewidth and actively recovers the sensing resolution. In this paper, we present the modal analysis, fabrication and characterization of the NS nanolaser. The small $V_{\mathrm{m}}$ and mode localization are revealed from their lasing and sensing characteristics.

We calculated the cavity mode using finite-difference time-domain (FDTD) method with structural parameters set as follows: the slab index $n_{\text {slab }}=3.4$, lattice constant $a$ $=500 \mathrm{~nm}$, airhole diameter $2 r=260 \mathrm{~nm}$, airhole shift $s_{\mathrm{x}}$ $=80 \mathrm{~nm}$, slab thickness $t=200 \mathrm{~nm}$, and NS width $w_{\mathrm{NS}}$ $=20 \mathrm{~nm}$. When air is assumed as the environmental medium, the calculated modal energy $\varepsilon\left|\boldsymbol{E}^{2}\right|$ is strongly localized in the NS, as shown in Fig. 1(a). Around the NS, the electric field vector $\boldsymbol{E}$ mainly orients normal to the sidewalls of the NS. Since the normal component of the displacement vector $\boldsymbol{D}$ is continuous across the boundary, $\varepsilon|\boldsymbol{E}|^{2}$ could be enhanced in the air region inside the NS to $\varepsilon_{\text {slab }}^{\prime} / \varepsilon_{\text {env }}$

\footnotetext{
${ }^{a)}$ Electronic mail: d09sd103@ynu.ac.jp.

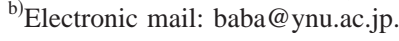

$=\left(n_{\text {slab }}^{\prime} / n_{\text {air }}\right)^{2}$ times, when $n_{\text {slab }}^{\prime}$ is the equivalent model index of the slab, and is calculated to be 2.6 in the present case. In the FDTD calculation, the enhancement was estimated to be 6.4 times, which is in good agreement with $\varepsilon_{\text {slab }}^{\prime} / \varepsilon_{\text {env }}$. However, when such devices are applied for biosensing, water is usually used as an environmental medium. We changed the environmental medium from air to water $\left(n_{\text {water }}=1.321\right)$ and calculated that the resonant wavelength redshifts from $\lambda$ $=1468$ to $1571 \mathrm{~nm}$ (from 1543 to $1612 \mathrm{~nm}$ without NS), $Q$ decreases from 2200 to 1400 (58 000 to 20100 ), and $V_{\mathrm{m}}$ increases from $0.028 \mu \mathrm{m}^{3}=0.0087\left(\lambda / n_{\text {air }}\right)^{3}$ to $0.035 \mu \mathrm{m}^{3}$ $=0.021\left(\lambda / n_{\text {water }}\right)\left(0.012\right.$ to $\left.0.015 \mu \mathrm{m}^{3}\right)$. The large redshift must be taken into account to match $\lambda$ with the gain peak of the laser wafer. The lower $Q$ and larger $V_{\mathrm{m}}$ in water suggest weaker mode confinement, but the $Q$ is still sufficiently high for laser operation.

To fabricate the device, we employ a GaInAsP/InP single quantum-well wafer with a gain peak at $\lambda=1.55 \mu \mathrm{m}$. The total thickness of GaInAsP layers, including the quantum well, is $180 \mathrm{~nm}$. We fabricate the device through $e$-beam lithography, hydrogen iodide (HI) inductively coupled plasma (ICP) etching of GaInAsP and $\mathrm{HCl}$ wet etching of InP. Using our $e$-beam writer Elionix ELS-7500 with positive resist Zeon ZEP520A, NS patterns of $w_{\mathrm{NS}}>20 \mathrm{~nm}$ is drawn simultaneously with airhole patterns of the PC without

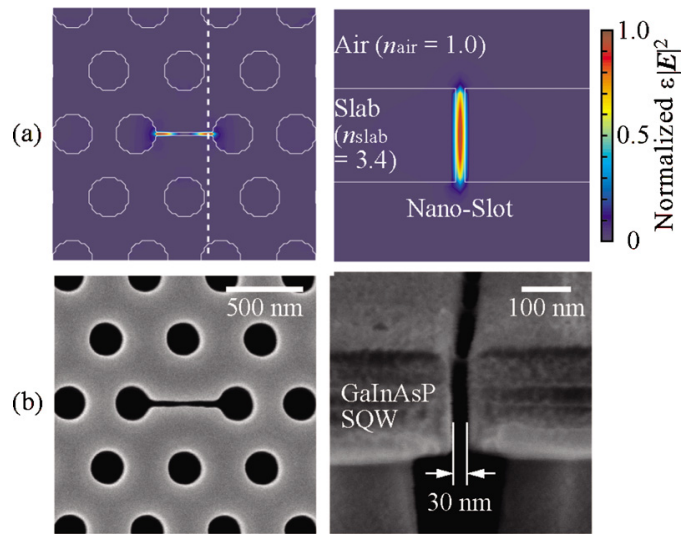

FIG. 1. (Color online) NS HO PC nanolaser (Left: top view, and right: cross-sectional view of NS). (a) Calculated modal energy distribution. (b) Fabricated device. 


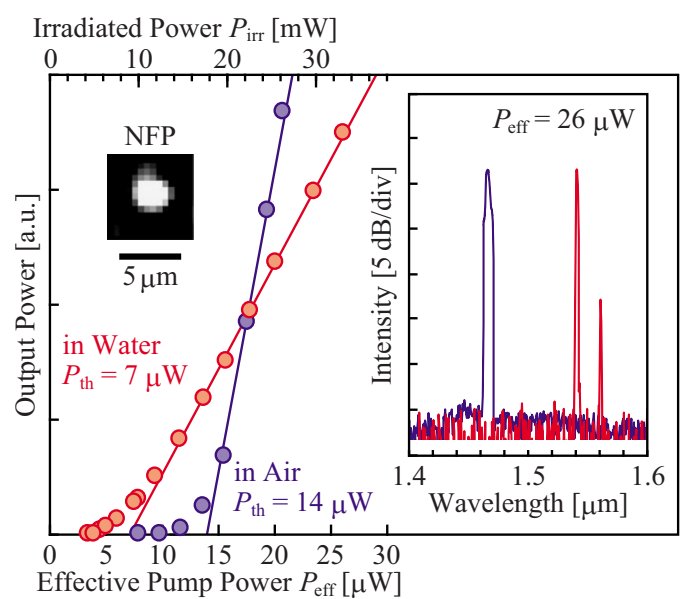

FIG. 2. (Color online) Laser characteristics in air (larger threshold) and water (smaller threshold). Left inset shows the near field pattern of light output in water. Right inset shows laser spectra in air (the shortest wavelength peak) and water (two peaks of longer wavelength side).

changing the resist thickness and exposure condition. In the HI ICP etching using Samco RIE-200ip, the lithography pattern is transferred to the wafer directly. ${ }^{11}$ The etching also achieves a high-aspect ratio of over 10, even for patterns smaller than $200 \mathrm{~nm}$ in size, and is particularly effective for the NS formation. Figure 1(b) shows scanning electron micrographs of the fabricated device, which shows that the NS is completely perforated. The smallest $w_{\mathrm{NS}}$ is $30 \mathrm{~nm}$, which is narrower than $60-150 \mathrm{~nm}$ reported so far for other NS devices. ${ }^{6-8}$

Optical characterization is performed by photo-pumping at room temperature under pulsed condition (175 ns, 10 $\mathrm{kHz}$ ). The localized mode in the NS is too small to pump exclusively. We rather noticed in the calculation that although the mode is mostly localized in the NS, it also extends out to the surrounding semiconductor at weak intensity. In fact, the mode is efficiently excited when the pump area is expanded to $25 \mu \mathrm{m}$ in diameter. Figure 2 shows the laser characteristics, where the effective pump power $P_{\text {eff }}$ is evaluated by considering the absorption and overlap efficiencies of the pump spot and the effective modal area. ${ }^{9}$ Clear operation is observed in both air and water with a threshold of $P_{\text {eff }}=14$ and $7 \mu \mathrm{W}$, respectively. Since the laser wavelength in water better matches the gain peak, the threshold is lower, although $Q$ is degraded by radiation loss as well as absorption loss in water $\left(\sim 20 \mathrm{~cm}^{-1}\right.$ at $\left.\left.\lambda=1.5 \mu \mathrm{m}\right)\right)^{12}$ The lower slope efficiency in water might be due to absorption loss and a lower collection efficiency of light output. The spectrum above threshold exhibits a peak intensity of $>25 \mathrm{~dB}$ higher than the noise level. The wide spectrum in air is caused by thermal chirping, as discussed further below. The multimodal behavior in water is also seen in the calculation. It might be caused by the break of symmetry in fabricated structures with some disordering.

To confirm the mode localization in the NS, we investigated the dependence of the laser characteristics on $w_{\text {NS }}$, as shown in Fig. 3. The experimental $\lambda$ (circular plots) varies with $w_{\mathrm{NS}}$ and exhibits a good agreement with the theoretical one (solid lines). Once the NS is introduced, $\lambda$ jumps to shorter wavelength, but changes little as $w_{\mathrm{NS}}$ is increased. Figure 4 shows the sensitivity to the environmental index $\Delta \lambda / \Delta n_{\text {env }}$, measured using index liquids $\left(n_{\text {env }}\right.$

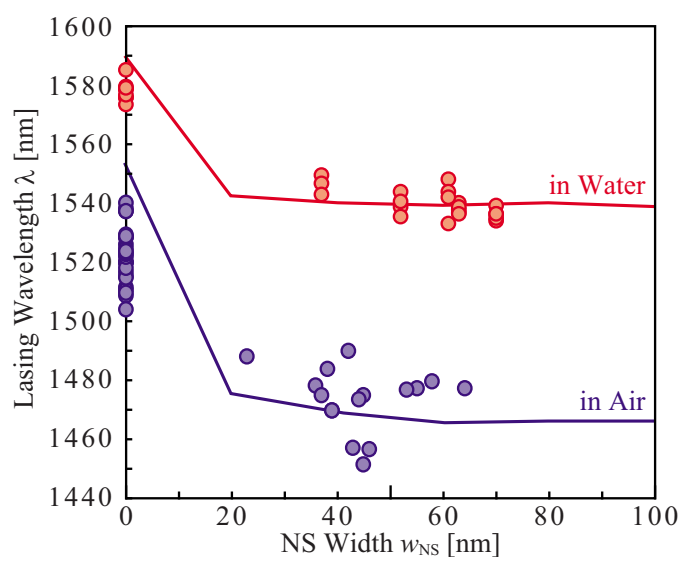

FIG. 3. (Color online) Dependence of laser wavelength $\lambda$ on NS width $w_{\mathrm{NS}}$ in air and water. Solid lines and circular plots show theoretical and experimental values, respectively.

$=1.296-1.344$ ). (a) compares the spectral shift in different liquids without and with the NS, clearly indicating that $\Delta \lambda / \Delta n_{\text {env }}$ is enhanced by the NS. (b) summarizes the dependence of $\Delta \lambda / \Delta n_{\text {env }}$ on $w_{\mathrm{NS}}$. A high sensitivity of 410 $\mathrm{nm}$ per refractive index unit (RIU) is obtained at $w_{\mathrm{NS}}$ $=75 \mathrm{~nm}$, which is the highest record for nanocavity-based sensors. The good agreement between the experimental and theoretical results shown here supports the hypothesis that the field penetration into liquids is enhanced by the NS. ${ }^{13}$ Figure 5(a) demonstrates the athermalization due to the NS; the temperature dependence $\Delta \lambda / \Delta T$ becomes 0.17 times with the NS at $T=20^{\circ} \mathrm{C}-30{ }^{\circ} \mathrm{C}$. Since water has a negative thermo-optic coefficient $\Delta n / \Delta T \sim-10^{-4} \mathrm{RIU} / \mathrm{K},{ }^{14}$ the positive coefficient $\sim 10^{4} \mathrm{RIU} / \mathrm{K}$ for GaInAsP slab can be canceled with appropriate mode penetration into the water. ${ }^{15}$ In addition, water has the quadratic temperature dependence, ${ }^{14}$ the cancelling becomes more efficient at higher temperature. The theoretical line taking into account these effects well explains experimental plots. The laser spectrum drastically changes between without and with the NS, as shown in Fig. 5(b). In pulsed lasing nanolasers, we often observe the spectral broadening due to very wide thermal chirping of up to 10 $\mathrm{nm}$ width; we confirmed this in the time-averaged and timeresolved measurement. ${ }^{16}$ In Fig. 5(b), however, the spectral linewidth for the NS device in water is as narrow as 18 p.m. (nearly the resolution limit of our spectrometer) thanks to the

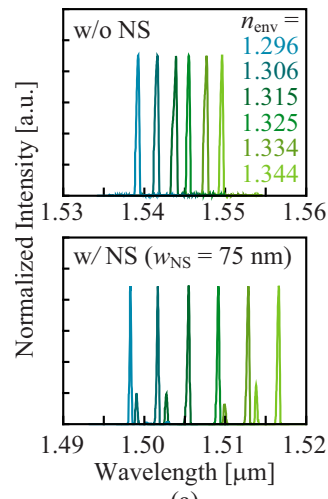

(a)

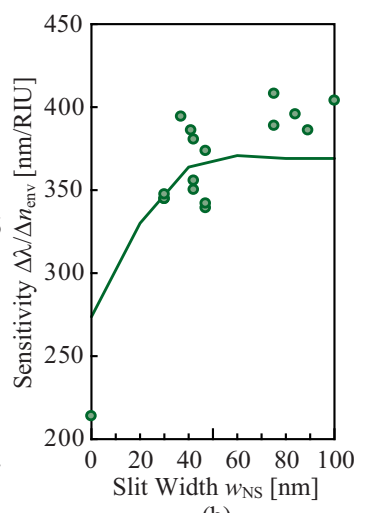

(b)
FIG. 4. (Color online) Index sensitivity. (a) Laser spectral shift in different index liquids without and with NS. (b) Dependence of $\Delta \lambda / \Delta n_{\mathrm{env}}$ on $w_{\mathrm{NS}}$. Solid lines and circular plots show theoretical and experimental values, respectively. 


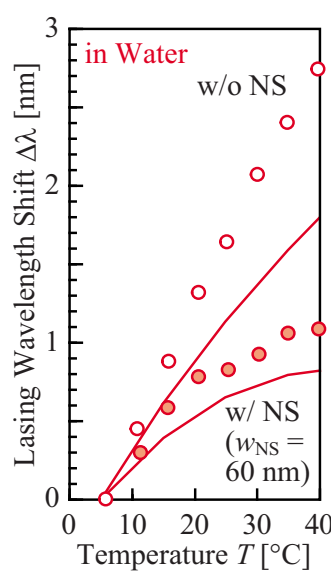

(a)

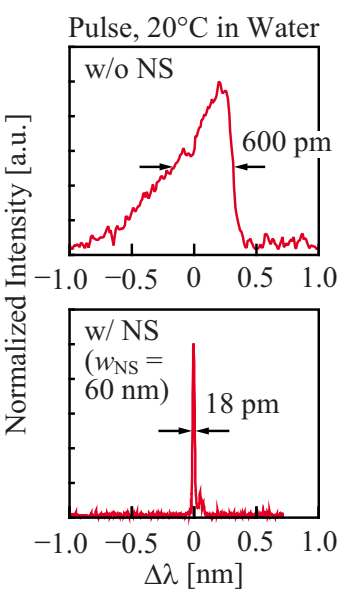

(b)
FIG. 5. (Color online) Temperature dependence of $\lambda$. (a) Wavelength shift $\Delta \lambda$ with device temperature $T$ in water without and with NS. Solid lines and circular plots show theoretical and experimental values, respectively. (b) Comparison of laser spectra in water without and with NS.

athermalization. From the enhanced sensitivity and reduced spectral linewidth, the index resolution is improved to 4.4 $\times 10^{-5}$ RIU, twice better than our previous one, ${ }^{10}$ while still being limited by the spectrometer.

In summary, we demonstrated the NS nanolaser with $w_{\mathrm{NS}}=30 \mathrm{~nm}$. The laser characteristics ensure that the experimental laser mode is strongly localized in the NS. Since it enhances the interaction of the cavity mode with the medium in the NS, one may apply this to cavity QEDs and nonlinear optics that use vapor atoms and molecules. The sensitivity against environmental index enhanced by the NS is advantageous for various sensing applications.

This work was supported by a Grant-in-Aid and a Research Fellowship of the Japan Society for the Promotion of Science.

${ }^{1}$ J. M. Gérard, B. Sermage, B. Gayral, B. Legrand, E. Costard, and V. Thierry-Mieg, Phys. Rev. Lett. 81, 1110 (1998).

${ }^{2}$ T. Baba, D. Sano, K. Nozaki, K. Inoshita, Y. Kuroki, and F. Koyama, Appl. Phys. Lett. 85, 3989 (2004).

${ }^{3}$ T. Yoshie, A. Scherer, J. Hendrickson, G. Khitrova, H. M. Gibbs, G. Rupper, C. Ell, O. B. Shchekin, and D. G. Deppe, Nature (London) 432, 200 (2004)

${ }^{4}$ X. D. Yang and C. W. Wong, Opt. Express 15, 4763 (2007).

${ }_{5}^{5}$ J. T. Robinson, C. Manolatou, L. Chen, and M. Lipson, Phys. Rev. Lett. 95, 143901 (2005).

${ }^{6}$ A. Di Falco, L. O'Faolain, and T. F. Krauss, Appl. Phys. Lett. 94, 063503 (2009).

${ }^{7}$ C. A. Barrios, M. J. Banuls, V. Gonzalez-Pedro Banuls, B. Sanchez, A. Griol, A. Maquieira, H. Sohlstrom, M. Holgado, and R. Casquel, Opt. Lett. 33, 708 (2008).

${ }^{8}$ A. H. J. Yang, S. D. Moore, B. S. Schmidt, M. Klug, M. Lipson, and D. Erickson, Nature (London) 457, 71 (2009).

${ }^{9}$ K. Nozaki, S. Kita, and T. Baba, Opt. Express 15, 7506 (2007).

${ }^{10}$ S. Kita, K. Nozaki, and T. Baba, Opt. Express 16, 8174 (2008).

${ }^{11}$ T. Ide, J. Hashimoto, K. Nozaki, E. Mizuta, and T. Baba, Jpn. J. Appl. Phys., Part 2 45, L102 (2006).

${ }^{12}$ K. F. Palmer and D. Williams, J. Opt. Soc. Am. 64, 1107 (1974).

${ }^{13}$ N. A. Mortensen, S. S. Xiao, and J. Pedersen, Microfluid. Nanofluid. 4, 117 (2008)

${ }^{14}$ J. Carper, Libr. J. 124, 10 (1999).

${ }^{15}$ C. Karnutsch, C. L. C. Smith, A. Graham, S. Tomljenovic-Hanic, R. McPhedran, B. J. Eggleton, L. O'Faolain, T. F. Krauss, S. Xiao, and N. A. Mortensen, Appl. Phys. Lett. 94, 231114 (2009).

${ }^{16} \mathrm{H}$. Watanabe, K. Nozaki, and T. Baba, Proceedings of the International Symposium on Compound Semiconductors, Kyoto, 2007. 